\title{
Uptake of Training on Vscan by Midlevel Providers Working in Rural Health Facilities in Tanzania: Implications for Reliability
}

Selemani Mbuyita ${ }^{1 *}$, Robert Tillya ${ }^{1}$, Ritha Godfrey ${ }^{1}$, Uzzell Janeen ${ }^{2}$, Kallol Mukherji' ${ }^{2}$ Amon Exavery ${ }^{1}$ and Godfrey Mbaruku ${ }^{1}$

${ }^{1}$ Ifakara Health Institute, P.O. Box 78373, Kiko Avenue, Mikocheni, Dar Es Salaam, Tanzania

${ }^{2} \mathrm{GE}$ Healthcare, 5 Roman Ridge, Accra, Ghana

\begin{abstract}
For decades now maternal mortality has remained high especially in developing countries. Reasons for these deaths are many but mainly due to health system failures such as critical shortages of health work force and limited use of appropriate technologies in service rendering among others.

New innovations are produced to reduce maternal mortality. One such innovation is the use of a hand held portable ultrasound called Vscan. However, while the Vscan is simplified to suit different contexts, availability of experts to manage and conduct scanning in rural health facilities is limited. Training of the available non clinician and midlevel health providers is one of the available options.

We conducted a study to ascertain if midlevel health providers working in rural settings in Tanzania can be trained and provide ultrasound scanning services using the Vscan in routine antenatal clinics.

We used a mix of training methods including presentations, lectures, group discussions, demonstration and hands on sessions to conduct the study.

Results showed that, despite the technology being sophisticated, the uptake of knowledge by the trainees was strongly satisfactory. Using the pre and post-tests method, there was a significant change in knowledge of the trainees when comparing their basic knowledge on radiology, sonology and pregnancy related complication detection and diagnosis that they had before training and after training. On the other hand, no statistical significant difference was noted in knowledge uptake and translating the knowledge in practice between the different cadres of the midlevel providers involved in the training. The quality of scanning using the Vscan between the trainees and those of expert sonologists was $77 \%$ compatible.
\end{abstract}

The study concludes that, it is possible to train health providers to conduct routine scanning using Vscan at primary health facility level and produce quality scans and correct diagnosis similar to that of expert sonologist.

Keywords: Training; Knowledge uptake; Vscan; Midlevel providers

\section{Introduction}

Between 2004 and 2010, Tanzania has managed to reduce maternal mortality from 578 to 454 per every 100,000 live births [1,2]. This slow reduction in maternal mortality does not prevail only in Tanzania but also in most of the developing countries worldwide. The low pace in reducing maternal mortality in developing countries has led to a realization that multiple strategies are required to address the problems at hand [3]. While health system interventions for maternal health across the continuum of care are being advocated, uses of technology in accelerating the pace at which maternal morbidity and mortality can be reduced are also being introduced [4]. The best example of simple but life-saving technology is the use of a simplified ultrasound device (called Vscan) for early detection of pregnancy complications. This technology helps women to develop effective birth plans and assist health providers to address the pregnancy related complications timely hence avoiding the adverse effect of the complications $[4,5]$.

On the other hand, introduction of sophisticated technologies in health services delivery faces a challenge of availability of skilled personnel to manage the technologies [5]. In many developing countries, health systems are severely constrained with critical shortages of health work force, not only in numbers but also in skills and qualifications. In many developing countries, skilled personnel to run services such as X-ray and Ultrasound are scarce [6]. In Tanzania for example, the proportion of radiologists is $0.2 \%$ out of the total number of health personnel in the country which is 56,196 . The total gap of radiologists in the country is as huge as $75 \%$ [7]. The situation is worse in the rural than in urban areas. The worst scenarios are seen in lower level facilities such as health centres and dispensaries. Majority of these lower level facilities are staffed with non-specialized staff most of whom are midlevel providers and junior nurses [6,7]. In such circumstances, the importance of investing on the existing human resources for health regardless of their previous training might be applicable as a transitional solution $[6,8]$. With the emerging technological innovations such as the Vscan, their wide spread use will depend on the existing health workforce. However, the investment should strike a balance between the need to spread such technological services and reliability of their use by the midlevel providers [5]. Quality of the services should not be compromised at the expense of haphazard investment on the existing health workforce $[5,6]$.

As a means to ensure balance between good quality and spread of

*Corresponding author: Selemani Mbuyita, Ifakara health Institute, P.O. Box 78373, Kiko Avenue, Mikocheni, Tanzania, Tel: +255 784653 222; Fax: +255 232333 487; E-mail: smbuyita@ihi.or.tz

Received June 19, 2014; Accepted November 11, 2014; Published November 25, 2014

Citation: Mbuyita S, Tillya R, Godfrey R, Janeen U, Mukherji K, et al. (2014) Uptake of Training on Vscan by Midlevel Providers Working in Rural Health Facilities in Tanzania: Implications for Reliability. J Biosafety Health Educ 2: 123. doi:10.4172/2332-0893.1000123

Copyright: ( 2014 Mbuyita S, et al. This is an open-access article distributed under the terms of the Creative Commons Attribution License, which permits unrestricted use, distribution, and reproduction in any medium, provided the original author and source are credited. 
Citation: Mbuyita S, Tillya R, Godfrey R, Janeen U, Mukherji K, et al. (2014) Uptake of Training on Vscan by Midlevel Providers Working in Rural Health Facilities in Tanzania: Implications for Reliability. J Biosafety Health Educ 2: 123. doi:10.4172/2332-0893.1000123

Page 2 of 5

\begin{tabular}{|c|c|c|c|c|c|c|}
\hline SN & Health Provider ID & Work station Level & Age (years) & Professional qualification & $\begin{array}{l}\text { Duration of pre-service } \\
\text { training (years) }\end{array}$ & $\begin{array}{c}\text { Duration in service } \\
\text { (years) }\end{array}$ \\
\hline 1. & ET_KIM1 & Dispensary & 27 & Assistant Clinical Officer & 2 & 2 \\
\hline 2. & ET_KIM2 & Dispensary & 43 & Nurse Midwife (NM) & 4 & 6 \\
\hline 3. & ET_HOM1 & Dispensary & 47 & Clinical Officer (CO) & 3 & 21 \\
\hline 4. & ET_HOM2 & Dispensary & 38 & Nurse Midwife (NM) & 3 & 2 \\
\hline 5. & ET_KUR2 & Dispensary & 33 & Nurse Assistant (NA) & 2 & 8 \\
\hline 6. & ET_KIH1 & Dispensary & 48 & Diploma in Clinical Medicine & 3 & 27 \\
\hline 7. & ET_KIH2 & Dispensary & 34 & Enrolled Nurse (EN) & 4 & 7 \\
\hline 8. & ET_GWA1 & Dispensary & 44 & Medical Attendant (MA) & 1 & 25 \\
\hline 9. & ET_GWA2 & Dispensary & 34 & Medical Attendant (MA) & 2 & 2 \\
\hline 10. & ET_MAS1 & Health Centre & 38 & Assistant Medical Officer (AMO) & 2 & 9 \\
\hline 11. & ET_MAS2 & Health Centre & 40 & Registered Nurse (RN) & 2 & 17 \\
\hline 12. & ET_MAS3 & Health Centre & 30 & Assistant Nurse Officer & 4 & 2 \\
\hline 13. & ET_KIS1 & Hospital & 34 & BSc Nursing & 3 & 11 \\
\hline 14. & ET_KIS2 & Hospital & 44 & $\mathrm{MCHA}$ & 2 & 24 \\
\hline
\end{tabular}

Table 1: Profiles of trainees.

life-saving services, on the job training methodologies that are effective enough to impart crucial knowledge but in a short period of time to personnel who would otherwise not be eligible to provide certain services are required [9]. Designing the training content, simplifying the complicated technical aspects of the new knowledge to be imparted, overcoming the language barrier especially when technologies are imported from manufacturers using foreign languages and selection of methods and approach of training are important training aspects that need a careful consideration [10].

We conducted a study to pilot the use of Vscan at a dispensary and health centre level in Kisarawe district, Tanzania between April 2012 and August 2013. The important component of the study was to determine the uptake of the training on use of Vscan by the midlevel providers who did not have any basic training on radiological assessment. This paper presents the process, content and outcomes of the training with an implication to the reliability of introducing the technology in a rural setting with similar context. The training aimed at imparting health care workers in primary health care level with knowledge and skills on Basic Obstetric Ultrasound scanning and ultimately enables them to use the Vscan technology in antenatal clinic for early detection of pregnancy related complications. Currently, ultrasound scanning in Tanzanian health system is happening only in district hospitals and above. The study intended to demonstrate if the technology can be introduced at a dispensary and health centre levels where the majority of women in the country seek reproductive health services.

\section{Methods}

\section{Study area and population}

This study was conducted in Kisarawe district, one of six rural districts in the Coast Region of Tanzania. Purposefully, ten health facilities were selected for the whole study based on volume of utilization of their respectful Ante natal clinics with the expectations of obtaining sufficient sample size of women to be involved in the study. Using a before and after design, five of the facilities (including their catchment communities) were used as intervention facilities while the remaining five were used as control facilities. In each arm, four dispensaries and one health centre were selected. The primary targeted audience was women of reproductive age who would benefit with introduction of ultrasound scanning in routine antenatal clinic at primary facility level. However, under the training component, target participants were health providers providing reproductive and child health services from the five intervention facilities.

\section{Selection of trainees}

Health providers directly involved in providing routine ante natal care (ANC) services were targeted in this training regardless of their academic background and specialization. However, for supervisory purposes, the facility in-charge of every selected health facility was also included in the training. While the selection of health facilities was randomly done, selection of health providers was purposefully done targeting only those rendering reproductive and child health services in the selected facilities.

A total of 14 health providers were selected for training. Two health workers were selected from each of the intervention dispensaries while three health workers were from a health centre. Two health providers from the district hospital were also selected for referral and supervisory purposes.

The professional cadres of the trainees included clinicians and different types of nurses. Table 1 provides profiles of all trainees participated in the training.

\section{Training methods and approach}

Adult learning approach was used with two broad categories of the training namely didactic and practical sessions. During the didactic sessions, methods of training included power point presentation, short lecture discussion, and group discussion and question and answer sessions. During the practical sessions, methods of training included demonstrations using models and sample images and hands on practical sessions.

\section{Training process}

Three hour morning sessions were used for presentations, short lecture discussions, demonstration and review of sample images. Power point was used to display short notes and sample images specific to the topic being discussed. Special models to represent pregnant woman was used to demonstrate different positions of a human body and anatomy. Flashlights represented ultrasound probe and was used to demonstrate how to hold and use the probe to identify different structures in a woman's abdomen.

Two teams of facilitators worked in a co - facilitation method. Three foreign expert facilitators (one from Hands for Humanity and two from GE Healthcare - all from the United States of America) and two local facilitators (from Muhimbili National Hospital) conducted the training. The medium of instruction was English but for elaborations 
Citation: Mbuyita S, Tillya R, Godfrey R, Janeen U, Mukherji K, et al. (2014) Uptake of Training on Vscan by Midlevel Providers Working in Rural Health Facilities in Tanzania: Implications for Reliability. J Biosafety Health Educ 2: 123. doi:10.4172/2332-0893.1000123

and clarifications, the local facilitators took role of interpreters and translators into Kiswahili to enable the trainees to better understand and comprehend some concepts.

Prior to the core training, trainees were subjected to a pre-test to assess their knowledge in pregnancy related complications and basic understanding of radiology in general and sonography in particular. At the end of the training, the same test was again subjected to trainees to measure the trainees' immediate uptake of the training. Refresher training was later conducted after the trainees have practiced in their respective health facilities for six months.

During practical training, all trainees were able to conduct scanning to at least two women each day for 10 days of the indoor training. While the theory part was being conducted in a classroom, the practical sessions were held in a special room prepared for training within the Kisarawe district hospital. Women drawn from Kisarawe district hospital ANC clinic were asked for their consent and those who consented were scanned by the trainees under the supervision of the trainers.

\section{Training content}

Participants were introduced to basics of sonography which included learning about the strength of the echo; white/ bright means strong reflector and represent the bone, grey medium reflector representing tissues and dark/black representing water/fluid. Together with general anatomy of woman's reproductive organs this was provided as prerequisite information to the hands on practical ultrasound training.

The participants were first oriented to the structure and function of the VScan device to be able to operate it. In this, they were taught on different types of transducers, how to use and take care of them. They were oriented to Brightness Mode (B-mode) to be able to increase or decrease light intensity to improve the quality of the image. The Color Doppler function was introduced for the health providers to be able to identify blood vessels such as in the cord in order to rule out abnormalities like cord presentation or cord around the neck. M-Mode to detect motion which helps to show the viability of the fetus, to increase or decrease the depth of scanning depending on the type and size of the image. The use of appropriate buttons on the device to increase/ decrease the frequency to change the resolution, increase or decrease the depth to display deep or shallow anatomy were all demonstrated.

Every day, prior to the start of the practical training, the health providers reviewed step by step, the technique and skills of scanning a pregnant woman's abdomen. This started from scanning a first trimester where participants learned how to use portable ultrasound to identify the uterus, urinary bladder, intra and extra uterine pregnancy, gestational and yolk sac and estimating fetal gestational age using crown rump length (CRL).

For the second trimester, they learned to estimate fetal gestation age using biparietal diameter (BPD) or femur length (FL) with reference from a physical chart. They also learned how to identify placenta previa and low lying placenta.

\section{Statistical analysis}

Analysis of data generated from training was generally descriptive, with frequency distribution of the respondents across variables calculated. For each of the professional cadres assessed, the proportion of providers with knowledge on ultrasound before and after training was recorded. Then the percentage change in the level of knowledge before and after training was calculated by subtracting the former from the latter. Due to a small sample size, error bars were used to determine whether or not the change/difference observed was statistically significant. For the data generated from health providers' practices when producing images from clients, normal frequency distribution across variables was calculated and two-sided paired t-test used to generate statistical significance.

\section{Results}

Of the 14 trainees, 13 trainees completed the training successfully. From practical sessions, independent assessment was done by the five trainers and 13 of the 14 trainees were confirmed to be able and trusted to carry out the task of undertaking scanning in routine ANC clinics in their respective work stations. One trainee showed unsatisfactory performance during the training which was associated more with his focus and paying attention to the training rather than difficulty of the training package or methodology.

The pre and post training tests showed a very big change in acquisition of knowledge on pregnancy related complications as well as on sonological practices. Using the error bars, this change was found to be statistically significant. Table 2 summarizes results of pre and post tests used as a measure of determining knowledge uptake by the trainees as an outcome of the training.

The minimum score during pre-test was $5 \%$ and the maximum

\begin{tabular}{|c|c|c|c|c|c|}
\hline Professional Cadre & Category of Profession & Rank of cadre by seniority & Pre-test results (\%) & Post-test Results (\%) & \%change \\
\hline Nurse Officer (NO) & Nurse & 1 & 25 & 85 & 60 \\
\hline Assistant Medical Officer (AMO) & Clinician & 2 & 25 & 95 & 70 \\
\hline Assistant Nurse Officer (ANO) & Nurse & 3 & 50 & 75 & 25 \\
\hline Assistant Nurse Officer (ANO) & Nurse & 3 & 40 & 90 & 50 \\
\hline Clinical Officer (CO) & Clinician & 4 & 10 & 45 & 35 \\
\hline Clinical Officer (CO) & Clinician & 4 & 10 & 75 & 65 \\
\hline Assistant Clinical Officer (ACO) & Clinician & 5 & 45 & 60 & 15 \\
\hline Nurse Midwife (NM) & Nurse & 6 & 25 & 70 & 45 \\
\hline Nurse Midwife (NM) & Nurse & 6 & 5 & 50 & 45 \\
\hline Nurse Midwife (NM) & Nurse & 6 & 10 & 80 & 70 \\
\hline Mother and Child Health Aide (MCHA) & Nurse & 7 & 25 & 50 & 25 \\
\hline Medical Attendant (MA) & Nurse & 8 & 10 & 55 & 45 \\
\hline Medical Attendant (MA) & Nurse & 8 & 5 & 65 & 60 \\
\hline Medical Attendant (MA) & Nurse & 8 & 20 & 70 & 50 \\
\hline
\end{tabular}

Table 2: Change in health providers' knowledge on ultrasound measured by a pre and post training test results. 
score was $50 \%$. During the post test, the minimum score was $45 \%$ and the maximum score was $95 \%$. The minimum percentage change in knowledge was $15 \%$ and the maximum was $70 \%$. The results also showed that there was no any significant difference among the different cadres of the trainees in terms of knowledge uptake ( $\mathrm{p}=0.4745$ for pretest and $\mathrm{p}=0.3182$ for post-test).

The results from the first three months scans by the providers in their working stations before the refresher training showed that $77 \%$ $(n=104)$ of the images from the scans by the trained health providers had good clarity and correct diagnosis that matches that of expert sonologists diagnosis. At the end of the study the correct diagnosis increased to $80.6 \%$. About $88 \%$ of the remaining $23 \%$ of the diagnosis where the assessment did not match that of the sonologists, when analysed by a third independent senior radiologist, it was seen that the midlevel providers were right in their assessment.

\section{Discussion}

On the job training has for many years been the only effective way to communicate new innovations and knowledge to staff who are already at work [10]. While such new innovations can be integrated into training curriculum in schools, such measures benefit only those who are going to get trained as pre-service trainees. Short term courses with modules encompassing packages of new knowledge will continue to serve as the main means of training and updating the already inservice staff [11].

However, while these trainings are useful, the approach, process, methods and packaging of the content are very important to bring out the desired training outcomes $[10,11]$. In a context like the one the Tanzania health sector is having where about two thirds of the existing workforce are midlevel providers [7], introduction of sophisticated packages of knowledge and skills like ultrasound scanning require careful planning. This is because, specialization is less practised in many developing countries [12] and health providers have in many cases found themselves train and practise matters beyond their basic pre-service training and in some cases become the specialists of many health fields at the same time $[6,13]$.

As such, simplicity of the otherwise complicated subject matters and content of the training is very important $[10,11]$. Results from this study confirm that choice of the medium of instruction, selection of right training methods and especially using adult learning methods are intrinsic to attain training outcome that can promise sustenance.

For many years, sonology has remained an area with few specialists. In Tanzania for example, the proportion of health providers who are in the radiology and sonology speciality constitutes less than $0.2 \%$ [7]. The complexity of the technology and high costs of ultrasound machines have contributed to the small numbers of these personnel. Unfortunately the importance of the sonological services has remained highly significant as always. In these decades when efforts to attain MDG 4 and 5 are at the top of the agenda, the importance of this speciality has increased. Several studies have documented the contribution of early detection of pregnancy related complications to save women lives through improved pregnancy management and better birth plans $[4,5]$.

With the Vscan innovation, both the complexity of the technology and cost barriers has been overcome. It reduces the need for highly specialized personnel to perform scanning. However, the technology can easily spread to lower levels of health facilities only if on the job training of whoever is available at the severely constrained staffing situation can demonstrate reliability [14].
Our study findings provide evidence of such reliability. The training process itself whereas 13 out of 14 trainees were able to finish the training and be confirmed by the experts to be able to perform the ultrasound scanning showed its reliability. In addition, the knowledge gain demonstrated by the pre and post-tests where in a statistical significant difference in knowledge change between before and after training was noted offers another measure of reliability of the training process, approach, methods and content packaging. Similar achievements had previously been recorded in a study conducted in Seattle where five trained midwives had all successfully achieved the desired training outcome and were confirmed by expert sonologists to reliably be able to conduct quality ultrasound scanning [14].

The study has also helped to confirm that, the simplicity of the technology offers a big solution of human resources crisis in developing countries that would need to introduce routine use of the Vscan. This is because; the study results showed that, the technology can correctly be used by even the lowest cadre of health providers [4,5,14]. In Tanzanian context where Medical Attendants are the lowest cadre but constituting the highest percentage (33\%) of all health workforce in the country [7], their participation in this training showed no any statistical difference in knowledge gain during training or correct diagnosis during use of the Vscan compared to higher cadres such as nursing officers or Assistant Medical Officers. In such contexts and with the type of trainees being of lower qualifications, packaging the training content in a manner that makes the training modules and topics simple and chronologically sound are important [15]. Other studies have also recorded this fact that with experience, the lower cadres can deliver equal levels of quality care as that provided by higher cadres $[12,13]$.

The training methods have also helped to register the recorded success in uptake of knowledge in the referred training. Trainers worldwide have emphasized the importance of method mix in training [16]. While the verbal presentation using power point slides, blackboards and flip charts have remained popular for decades, limiting some of the lectures and turn them into exercises for group discussion has shown to improve training outcomes $[16,17]$. In addition, augmenting on the job training with actual hands on sessions helps to translate the theory part of the training into practice $[18,19]$.

\section{Conclusion}

This study provides evidence that, it is possible to train health providers to conduct routine scanning using Vscan at primary health facility level and produce quality scans and correct diagnosis similar to that of expert sonologist. It also offers evidence to show that, midlevel providers regardless of their cadres can equally produce the same quality of ultrasound services regardless of their pre-service training. The combination of well-designed training content, simplicity of the technology, right mix of the training methods and the whole process used in the training was fundamental to the high level of knowledge uptake demonstrated by the trainees during the Kisarawe district Vscan training. The training content and process can thus be produced into a training kit for use in similar settings within and outside the country.

\section{Acknowledgement}

The list of those who deserve to be acknowledged following completion of the work that leads to this publication is long. The authors would like to acknowledge all of them in a general statement and that their contribution during the project design and set up, approval, implementation, data collection, data processing and drafting the manuscript is very invaluable. Out of the long list, we would like to name a few due to their specific and vital contributions. These include the District Executive Director of Kisarawe District Council, District Medical Officer of Kisarawe district, The Medical Doctor in-charge of Kisarawe District hospital and 
Citation: Mbuyita S, Tillya R, Godfrey R, Janeen U, Mukherji K, et al. (2014) Uptake of Training on Vscan by Midlevel Providers Working in Rural Health Facilities in Tanzania: Implications for Reliability. J Biosafety Health Educ 2: 123. doi:10.4172/2332-0893.1000123

Page 5 of 5

The District Reproductive and Child Health Coordinator. We also acknowledge all health providers from the health facilities that were involved in the project. This work was supported by GE Healthcare East Africa Services Limited [Grant number MWO/9292998]

\section{Declaration of Conflicting Interests}

Two authors, namely Uzzell Janeen and Kallol Mukherji work for the GE Healthcare which was also the funding agency for the project. GE Healthcare is also the manufacturer of the Vscan device used during the project. However, the participation of the GE Healthcare was tied neither to any commercial benefits nor to individual gains. Their contribution to the development of this manuscript was in the real sense of reviewing and commenting across different sections of the manuscript.

\section{Research Ethics}

This work received ethical approval from the Ifakara Health Institute Institutional Review Board (IHI - IRB) - certificate number IHI/IRB/No.35. The IRB is registered with FEDERAL WIDE ASSURANCE NUMBER: SWA 00002632.

\section{References}

1. National Bureau of Statistics and ICF Macro (2004). Tanzania Demographic Health Survey. United Republic of Tanzania.

2. National Bureau of Statistics and ICF Macro (2011) Tanzania Demographic Health Survey. United Republic of Tanzania.

3. Ruth Simons, Peter Fajans and Laura Ghiron (2007) Scaling up health service delivery; from pilot innovations to policies and programmes. World Health organization.

4. Robert Harris D, William Marks M (2009) Compact Ultrasound for Improving Maternal and Perinatal Care in Low Resource Setting. Review of Potential Benefits, Implementation Challenges and Public Health Issues. J Ultrasound Med 28: 1067-1076.

5. Bret Nelson P, Edward Melnick R, James Li (2009) Portable Ultrasound for Remote Environments: Part I. Feasibility for Field Deployment. J Ultrasound Med 40: 190-197.

6. Maman Dogba, Pierre Fournier (2009) Human Resources and Quality of Obstetric Care in Developing Countries: a systematic Review of the Literature. Human Resources for Health 7:7.

7. United Republic of Tanzania. Ministry of Health and social Welfare (2013). Human Resource for Health Strategic Plan 2014-2019.
8. World Health Organization (2009). Increasing Access to Health Workers in Remote and Rural Areas through Improved Retention. Health Systems and Services Cluster (HSS); Human Resources for Health Department $(\mathrm{HRH})$ Health Workforce Migration and Retention Unit (HMR): Background paper.

9. Dieleman M, Cuong PV, Anh LV, Martineau T (2003) Identifying factors for job motivation of rural health workers in North Viet Nam. Hum Resour Health 1: 10

10. Beach MC, Price EG, Gary TL, Robinson KA, Gozu A, et al. (2005) Cultural competence: a systematic review of health care provider educational interventions. Med Care 43: 356-373.

11. Kurt Kraiger J, Kevin Ford, Eduardo Salas (1993) Application of Cognitive Skill-Based and Affective Theories of Learning Outcomes to New Methods of Training Evaluation. JAP. 78: 311-328.

12. Fulton BD, Scheffler RM, Sparkes SP, Auh EY, Vujicic M, et al. (2011) Health workforce skill mix and task shifting in low income countries: a review of recent evidence. Hum Resour Health 9: 1.

13. Hongoro C, McPake B (2004) How to bridge the gap in human resources for health. Lancet 364: 1451-1456.

14. Waylon Brunette, Wayne Gerard, A. Matthew et al. (2010). Portable Antenatal Ultrasound Platform for Village Midwives.

15. Kimberly HH, Murray A, Mennicke M, Liteplo A, Lew J, et al. (2010) Focused maternal ultrasound by midwives in rural Zambia. Ultrasound Med Biol 36 : 1267-1272.

16. Werner D, Bower B (2005) Helping health workers learn: a book of methods, aids, and ideas for instructors at the village level. pp 6001- 617.

17. Rowe AK, de Savigny D, Lanata CF, Victora CG (2005) How can we achieve and maintain high-quality performance of health workers in low-resource settings? Lancet 366: 1026-1035.

18. Dovlo D (2004) Using mid-level cadres as substitutes for internationally mobile health professionals in Africa. A desk review. Hum Resour Health 2: 7.

19. Lewin S, Munabi-Babigumira S, Glenton C, Daniels K, Bosch-Capblanch $X$, et al. (2010) Lay health workers in primary and community health care for maternal and child health and the management of infectious diseases. Cochrane Database Syst Rev: CD004015. 